\title{
Educação em saúde para o combate de enteroparasitoses em crianças de uma creche filantrópica em Belém - PA: relato de experiência
}

\author{
Health education to combat enteroparasitosis in child day care center in Belém - PA: \\ experience report
}
Educación de salud para combatir la enteroparasitosis en niños de um Jardines infantiles en Belém - PA: informe de experiência

Thasmyr das Mercês Gonçalves Corrêa1, Taynara Rodrigues Araujo, José Eduardo Gomes Arruda ${ }^{1 *}$.

\section{RESUMO}

Objetivo: Desenvolver atividades de educação em saúde, visando o combate as enteroparasitoses em crianças atendidas em uma creche filantrópica em Belém-PA. Relato de experiência: $O$ estudo foi desenvolvido na creche, no período de abril a novembro de 2019, com aproximadamente 170 crianças de ambos os gêneros e faixa etária de 2 a 7 anos, que viviam em condições de vulnerabilidade social. Foram desenvolvidas e aplicadas estratégias de educação em saúde junto as crianças, seus familiares e colaboradores da creche, com o intuito de controlar e prevenir as doenças parasitárias na população atendida. Houve grande interesse e participação das crianças e dos colaboradores da creche, em todas as atividades desenvolvidas. Para que o processo educativo se concretize positivamente, é preciso que os familiares e colaboradores reforcem as orientações dadas e deem disponibilidade às crianças de discutirem a respeito. Considerações finais: Diante disso, novos estudos devem ser executados em populações semelhantes, buscando o controle de enteroparasitoses em crianças atendidas em ambientes de creches. Por fim, salientase a importância das atividades de educação em saúde na promoção da saúde na comunidade e contribuindo simultaneamente com a formação ética dos futuros profissionais.

Palavras-chave: Educação em saúde, Doenças parasitárias, Creches.

\begin{abstract}
Objective: To develop health education activities, aiming to combat enteroparasitosis in children attended at a philanthropic daycare center in Belém-PA. Experience report: The study was carried out in the nursery, from April to November 2019, with approximately 170 children of both genders and age group from 02 to 07 years old, who lived in vulnerable conditions Social. Health education strategies were developed and applied to children, their families and daycare staff, in order to control and prevent parasitic diseases in the population served. There was great interest and participation by the children and the day care center employees, in all activities developed. For the educational process to materialize positively, it is necessary that family members and collaborators reinforce the guidelines given and make children available to discuss it. Final considerations: In view of this, new studies should be carried out in similar populations, seeking to control enteroparasitosis in children cared for in daycare settings. Finally, it highlights the importance of health education activities in promoting health in the community and simultaneously contributing to the ethical training of future professionals.
\end{abstract}

Keywords: Health education, Parasitic diseases, Child day care centers

1 Universidade Federal do Pará, Belém - PA. *E-mail: josearruda@ufpa.br

SUBMETIDO EM: 5/2020

ACEITO EM: 5/2020

PUBLICADO EM: 9/2020 


\section{RESUMEN}

Objetivo: Desarrollar actividades de educación para la salud, con el objetivo de combatir la enteroparasitosis en niños atendidos en una guardería filantrópica en Belém-PA. Informe de experiencia: El estudio se realizó en la guardería, de abril a noviembre de 2019, con aproximadamente 170 niños de ambos sexos y grupos de edad de 02 a 07 años, que vivían en condiciones vulnerables social Se desarrollaron estrategias de educación para la salud y se aplicaron a los niños, sus familias y al personal de guardería, para controlar y prevenir las enfermedades parasitarias en la población atendida. Hubo gran interés y participación de los niños y los empleados de la guardería en todas las actividades desarrolladas. Para que el proceso educativo se materialice positivamente, es necesario que los miembros de la familia y los colaboradores refuercen las orientaciones dadas y hagan que los niños estén disponibles para discutirlo. Consideraciones finales: En vista de esto, nuevos estudios deben llevarse a cabo en poblaciones similares, buscando controlar la enteroparasitosis en niños atendidos en guarderías. Finalmente, destaca la importancia de las actividades de educación para la salud en la promoción de la salud en la comunidad y, al mismo tiempo, contribuye a la formación ética de los futuros profesionales.

Palabras clave: Educación en salud, Enfermedades parasitarias, Jardines infantiles

\section{INTRODUÇÃO}

As parasitoses intestinais apresentam uma elevada prevalência, principalmente em países em desenvolvimento, onde são responsáveis por graves problemas de saúde com consequências sociais e econômicas. Apesar de bastante frequentes no Brasil, estas doenças são consideradas negligenciadas, com a real frequência ainda desconhecida na Amazônia legal (KUNZ JMO, et al., 2008; SEGER J, et al., 2010).

As doenças parasitárias são frequentes em crianças em idade escolar, principalmente nas que pertencem às classes socioeconômicas mais baixas. Ausência de conhecimento sobre hábitos de higiene, falta de saneamento básico e elevado período de permanência em escolas e creches, são os principais fatores relacionados as infecções nessa faixa etária. A transmissão destes patógenos ocorre principalmente pela ingestão de água e/ou alimentos contaminados ou contato da pele e mucosas com larvas (GOMES RP, et al., 2006; BIASI LA, et al., 2009; BISCEGLI TS, et al., 2009; LUDWING KM, et al., 2012).

Tais parasitos alteram o estado nutricional do hospedeiro, afetando principalmente a digestão e absorção dos nutrientes, podendo ocasionar diarreia crônica, má absorção, anemia ferropriva, baixa capacidade de concentração e dificuldades no aprendizado. A presença de parasitos intestinais que espoliam o organismo pela ingestão de sangue, como Ancylostoma duodenale e Necator americanus pode, em indivíduos resultar em anemia. No entanto, a anemia pode ser dependente da intensidade da infecção e de fatores nutricionais específicos da população estudada, bem como das reservas de ferro do hospedeiro. Infecções por $A$. lumbricoides podem estar relacionadas à anemia por prejuízo na absorção de nutrientes (FERREIRA UM, et al, 2000; KUNZ JMO, et al., 2008; SMITH JL e BROOKER S, 2010).

A maioria das parasitoses intestinais é bem tolerada pelo hospedeiro imunocompetente, cursando de forma assintomática, as crianças parasitadas, podem apresentar interferência no desenvolvimento cognitivo e físico, alterações psicossociais e até óbitos. Essas complicações podem comprometer a capacidade de atenção e o rendimento escolar, dificultando o aprendizado e o desenvolvimento nutricional das mesmas (FERNANDES S, et al, 2012).

Atualmente, o ambiente de creche é uma realidade na vida de muitas crianças. É o local onde passam a maior parte do dia e, com isso, fica claro o importante papel que essa instituição tem no desenvolvimento integral delas em seus aspectos físico, psicológico, intelectual e social. Uma creche adequada é capaz de favorecer a prevenção e o diagnóstico precoce de alguns problemas de saúde, além de estimular as crianças em suas diversas etapas do desenvolvimento (ORO D, et al., 2012). 
Em crianças que frequentam creches, o ambiente coletivo pode proporcionar maior potencial de transmissão de doenças através do contato interpessoal, treinamento inadequado de funcionários e deficientes condições de higiene. Além disso, as crianças neste estágio de vida apresentam imaturidade do sistema imunológico e hábitos de higiene em formação. Tais fatores podem elevar o risco de contaminação por agentes infecciosos (BELLOTO MVT, et al. 2011).

Mesmo com os avanços das políticas públicas nos últimos anos, as populações mais carentes continuam tendo menor acesso aos serviços de saúde. A educação em saúde é uma ferramenta importante na promoção da saúde e prevenção das parasitoses intestinais. Além das ações de conscientização em saúde, investimentos em saneamento básico são necessários para o controle destes patógenos. Diante disto, este estudo propôs desenvolver atividades de educação em saúde, visando o combate às enteroparasitoses em crianças atendidas em uma creche filantrópica em Belém-PA.

\section{RELATO DE EXPERIÊNCIA}

O estudo foi desenvolvido na creche filantrópica no período de abril a novembro de 2019. Durante o estudo, eram atendidas na creche, cerca de 170 crianças de ambos os gêneros e faixa etária de 02 a 07 anos, que viviam em condições de vulnerabilidade social. As crianças atendidas, permaneciam de 07 às 18 horas, de segunda a sexta-feira. Possuíam aula no período da manhã e executavam 3 (três) refeições diárias na mesma. As atividades de educação em saúde, foram desenvolvidas semanalmente, no horário de 08:00 às 12:00, por duas alunas de extensão e um professor coordenador, todos do curso de farmácia da Universidade Federal do Pará.

Como primeira atividade executada, foi criado um grupo de discussão, com a participação das alunas, do professor coordenador e os colaboradores da creche. O objetivo foi identificar o grau de conhecimento dos colaboradores, acerca das enteroparasitoses e identificar quais os hábitos de higiene eram comumente executados. A partir daí, foram estruturados treinamentos teóricos e acompanhamento deles.

As crianças da creche foram divididas em grupos menores, de no máximo 20, onde cada uma, participou de pelo menos, 7 atividades ao longo do projeto.

Para as crianças de 2 a 4 anos, o foco das atividades, foi à higiene pessoal. As crianças participaram de uma roda de conversa com explicações simples e linguagem de fácil acesso, onde foram indagadas sobre os cuidados com a lavagem das mãos, banho, escovação dentária e cuidado com os alimentos. Após o relato, a forma correta da assepsia das mãos e escovação dentária, foi transmitida por meio de brincadeiras educativas, com atividades de pintura, músicas infantis e teatro com fantoches, a fim de facilitar a memorização e aprendizagem das crianças.

Para as crianças maiores de 4 anos, além da higiene pessoal, a promoção da alimentação saudável, a prevenção de enteroparasitoses foram desenvolvidas em atividades lúdicas e de fácil entendimento. Também foi utilizado uma roda de conversa onde as crianças foram indagadas sobre os assuntos propostos. Elas receberam orientações sobre higiene bucal e qual a maneira correta de escovar os dentes e utilização do fio dental, além da forma correta da assepsia das mãos.

Quanto à alimentação saudável, a pirâmide alimentar brasileira e os riscos de uma alimentação inadequada, foram apresentados utilizando rodas de conversa, vídeos educativos e na confecção de murais. As enteroparasitoses foram apresentadas através de teatro com fantoches, atividades de pintura e confecção de murais, onde foram apresentadas as formas de contágio, sintomatologia e prevenção de tais infecções.

Os colaboradores participaram de um treinamento ministrado pelas alunas de extensão, que teve como tema manipulação de alimentos e os cuidados necessários. O objetivo dessa intervenção foi destacar a importância nutricional dos alimentos, com a apresentação da pirâmide alimentar brasileira, os cuidados com a sua manipulação, dando um enfoque as medidas de higiene, como profilaxia das parasitoses intestinais e bem como a conservação dos alimentos manipulados. 
Os familiares das crianças atendidas participaram de duas palestras, ministradas pelas alunas de extensão, onde foram apresentadas informações sobre sinais e sintomas, medidas de prevenção individual e coletiva das doenças parasitárias mais frequentes. Após as palestras, os familiares eram convidados a participarem de grupos de discussão, onde questionaram sobre os assuntos tratados, além de diagnóstico e tratamento das doenças parasitárias. Durante os grupos de discussão, receberam material impresso com as mediadas de prevenção, e foram informados acerca das atividades desenvolvidas com as crianças.

\section{DISCUSSÃO}

Houve grande interesse e participação das crianças e dos colaboradores da creche, em todas as atividades desenvolvidas. Além disso, foram relatados à equipe do projeto de extensão, que ao chegarem a suas casas, as crianças contavam aos pais sobre as atividades desenvolvidas.

Durante a interação, as respostas das crianças às perguntas realizadas sobre hábitos de higiene, foram corretas na maioria das vezes, demonstrando que elas possuíam algum conhecimento sobre o assunto, todavia, aplicavam com pouca frequência tal conhecimento, fator que favorece a susceptibilidade para doenças infecciosas. Tal informação corrobora com o observado por Gomes IO, et al. (2017) em uma creche no município de Montes Claros - MG.

A atividade sobre alimentação saudável identificou um elevado consumo de açucares, doces e alimentos processados entre as crianças, além de pouco consumo de frutas, verduras e legumes. Os alimentos mais citados foram biscoitos, macarrão instantâneo, sucos artificiais de caixa, salgadinhos e refrigerantes. Tais alimentos faziam parte das refeições (café da manhã e lanche da tarde) disponibilizadas pela creche.

A nutrição adequada na criança é fundamental para garantir o seu crescimento e desenvolvimento de forma saudável, sendo este estágio de vida um dos biologicamente mais vulneráveis. Vários estudos indicam que houve um crescimento da prevalência de obesidade e de doenças crônicas não transmissíveis (DCNT) associadas à alimentação, na qual é chamado atenção para as taxas na população infantil (AIRES APP, et. al., 2011; SPARRENBERGER K, et. al., 2015).

De acordo com Ritchie B, et al. (2015) a infância é uma fase importante para prevenir as DCNT por meio do incentivo e da adoção de hábitos saudáveis que irão permanecer durante a fase adulta.

Quanto à atividade sobre enteroparasitoses, observou-se pouco conhecimento das crianças acerca deles, principalmente quanto as formas de infecção e prevenção. A sintomatologia mais relada durante as rodas de conversa foi a "dor de barriga", expressão utilizada tanto para cólica intestinal quanto para diarreia. Tal constatação é preocupante, pois as crianças atendidas possuíam situação socioeconômica favorável ao advento de doenças parasitárias. Além disso, as crianças são um grupo de alto risco para infecções, pois podem entrar em contato com estes desde poucos meses de vida (GURGEL RQ, et al., 2005).

A prevalência de enteroparasitos nesta população ainda é negligenciada em Belém-PA, Magalhães RF, et al. (2013) observaram prevalência de $36,6 \%$ de enteroparasitos em crianças de creches na região do Vale do Aço - MG, enquanto Silva AO, et al. (2014) observaram prevalência de 29,6\% em crianças das creches municipais de Itapuranga - GO e Antunes AS e Libardoni KSB (2017) observaram prevalência de $18 \%$ no município de Santo Ângelo - RS.

A rotina dentro da educação infantil, mais precisamente na creche, já é uma forma de preparo e entendimento da vida em sociedade, pois, a criança é levada a entender que ela vai adquirir uma rotina, que diariamente vai se organizando, ordenando suas formas de fazer e agir em meio ao ambiente e as relações sociais que a cercam (DESSEN MA e POLONIA AC, 2007).

A partir das atividades de educação em saúde com as crianças, percebeu-se que elas estavam bem mais empenhadas em querer conhecer e combater as enteroparasitoses, colocando em prática o que as foi ensinado. 
O treinamento sobre os cuidados na manipulação de alimentos mostrou-se bastante positivo. Houve grande interesse e participação dos colaboradores da creche, surgindo perguntas que foram esclarecidas pelas alunas de extensão. A atividade mostrou uma carência de informações dos colaboradores em relação à manipulação e conservação adequada dos alimentos. Tal carência de informações, também foi observado por Silva AVM, et al. (2016) em uma creche no município de Belo Horizonte - MG e pode ser um fator de risco para a transmissão de enteroparasitos, uma vez que manipuladores de alimentos parasitados e assintomáticos podem representar uma fonte de transmissão relevante.

Considerando que a maioria das infecções parasitárias é transmitida por alimentos e reconhecendo a relevância dos manipuladores de alimentos, devem-se considerar esses trabalhadores como potenciais transmissores de enteroparasitoses (SIMÕES J e ALEIXO DL, 2014).

Foram ministrados dois treinamentos em forma de palestra, aos familiares das crianças. $O$ foco dessa atividade foi apresentar as principais parasitoses intestinais, seus sinais e sintomas, formas de infecção e profilaxia delas. Além disso, o treinamento contou com informações sobre alimentação saudável e cuidados necessários com a ingestão de água. Informações sobre saneamento básico e higiene pessoal devem estar incluídas às atividades de educação em saúde para um maior controle das doenças parasitárias. A educação em saúde é fundamental pois contribui na melhoria dos hábitos de higiene da população, como a higiene das mãos e dos alimentos consumidos (TEIXEIRA PA, 2016).

Houve uma baixa adesão dos familiares das crianças aos treinamentos propostos. Menos de $10 \%$ dos pais e/ou responsáveis compareceram às duas palestras. $O$ desinteresse apresentado pode contribuir negativamente no processo de ensino em saúde, pois a participação dos responsáveis na educação favorece o processo de ensino-aprendizagem e contribui para a formação global das crianças (COSTA EL e SOUZA JRS, 2019).

Para que o processo educativo se concretize positivamente, é preciso que os familiares e colaboradores reforcem as orientações dadas e deem disponibilidade às crianças de discutirem a respeito. Diante disso, novos estudos devem ser executados em populações semelhantes, buscando o controle de enteroparasitoses em crianças atendidas em ambientes de creches. Por fim, salienta-se a importância das atividades de educação em saúde na promoção da saúde na comunidade e contribuindo simultaneamente com a formação ética dos futuros profissionais.

\section{FINANCIAMENTO}

O presente estudo recebeu apoio financeiro da Universidade Federal do Pará, por meio da Pró-Reitoria de Extensão (PROEX), conforme Edital PIBEX/PROEX № 01/2019.

\section{REFERÊNCIAS}

1. AIRES APP, et. al. Consumo de alimentos industrializados em pré-escolares. Rev. da AMRIGS, 2011; 55(4): 350 355.

2. ANTUNES AS E LIBARDONI KSB. Prevalência de enteroparasitoses em crianças de creches do município de Santo Ângelo, RS, Revista Contexto \& Saúde, 2017; 32(17): 144 - 156.

3. BELLOTO MVT, et al. Enteroparasitoses numa população de escolares da rede pública de ensino do Município de Mirassol, Rev Panamaz Saude, 2011; 2: 37 - 44.

4. BIASI LA, et al. Prevalência de Enteroparasitoses em crianças de entidade assistencial de Erechim/RS, Revista Perspectiva, 2010; 125(34): $173-179$.

5. BISCEGLI TS, et al. Estado nutricional e prevalência de Enteroparasitoses em crianças matriculadas em creche, Revista Paulista de Pediatria, 2009; 27(3): 289 - 295.

6. COSTA EL e SOUZA JRS. Família e escola: as contribuições da participação dos responsáveis na educação infantil. Revista Khora, 2019; 6(7): 1 - 17.

7. DESSEN MA e POLONIA AC. A Família e a Escola como contextos de desenvolvimento humano, Paidéia, 2007; $17(36): 21-32$. 
8. FERNANDES S, et al. Protocolo de parasitoses intestinais, Acta Pediátrica Portuguesa, 2012; $43(1): 35$ - 41.

9. FERREIRA UM, et al, Tendência secular das parasitoses intestinais na infância na cidade de São Paulo (1984-1996), Revista de Saúde Pública, 2000; 34(6): 73- 82.

10. GOMES RP, et al. Fatores condicionantes de parasitoses intestinais em crianças de 1 a 8 anos de idade, Trabalho de Conclusão de Curso Pós-Graduação Lato sensu - Academia de Ciência e Tecnologia de São José do Rio Preto SP, São José do Rio Preto, 2006; 39 p.

11. GOMES IO, et al. Promoção da higienização das mãos em crianças para a prevenção de verminoses intestinais: um relato de experiência, Revista Multitexto, 2017; 5(1): 24 - 27.

12. GURGEL RQ, et al. Creche: ambiente expositor ou protetor nas infestações por parasitos intestinais em Aracaju, SE, Rev Soc Bras Med Trop, 2005; 38(3): 267 - 9.

13. KUNZ JMO, et al. Parasitas intestinais em crianças de escola municipal de Florianópolis, SC - Educação ambiental e em saúde, Biotemas, 2008; 21(4): 157 - 62.

14. LUDWING KM, et al. Ocorrência de Enteroparasitoses na população de um bairro da cidade de Cândido Mota-SP, Revista do Instituto de Ciência da Saúde, 2012; 30(3): 271 - 276.

15. MAGALHÃES RF, et al. Ocorrência de Enteroparasitoses em Crianças de Creches na Região do Vale do Aço - MG, UNOPAR Cient Ciênc Biol Saúde, 2013; 15(3): 187-91.

16. ORO D, et al. Prevalência de parasitas intestinais em crianças de Descanso, Unoesc Ciência - ACBS, $2010 ; 2$ : 151 $-156$.

17. RITCHIE B, et al. In the Kitchen' impact evaluation: engaging primary school students in preparing fruit and vegetables for their own consumption, Health Promotion Journal of Australia, 2015; 26(2): 146 - 149.

18. SEGER J, et al. Prevalência de parasitas intestinais na população do Bairro Salete, município de São Miguel do Oeste, SC, Unoesc \& Ciência - ACBS, 2010;1(1): 53 - 6.

19. SILVA AO, et al. Epidemiologia e prevenção de parasitoses intestinais em crianças das creches municipais de Itapuranga - GO, Revista Faculdade Montes Belos (FMB), 2015; 8(1): 2 - 17.

20. SILVA AVM, et al. Ações de extensão para promoção da saúde em creche no município de Belo Horizonte, MG, Interagir: pensando a extensão, 2016; 22: 32 - 45.

21. SIMÕES J e ALEIXO DL. Prevalência de enteroparasitoses em manipuladores de alimentos de escolas municipais de Campo Mourão - Paraná, SaBios: Rev. Saúde e Biol., 2014; 9(1): 75 - 85.

22. SMITH JL e BROOKER S. Impact of hookworm infection and deworming on anaemia in non-pregnant populations: a systematic review, Trop. Med. Int. Health., 2010; 15(7): 776 - 795.

23. SPARRENBERGER K, et al. Ultra-processed food consumption in children from a Basic Health Unit, Jornal de Pediatria, 2015; 6: 535 - 542.

24. TEIXEIRA PA. Conhecimentos sobre parasitoses intestinais como estratégia para subsidiar ferramentas de educação em saúde. Dissertação (Mestrado em Ciências) - Instituto Oswaldo Cruz. Pós Graduação em Medicina Tropical, Rio de Janeiro, 2016; $81 \mathrm{p}$. 